\title{
Communication skills and counselling
}

\author{
Sunera M Fernando ${ }^{1}$ \\ Sri Lanka Journal of Obstetrics and Gynaecology 2011; 33: 69-71
}

\section{Introduction}

Counselling involves a process, the aim of which is to help persons to help themselves by making better choices. It supports people to make constructive changes in their lives. Counselling may be necessary to promote healthy choices. Patients may benefit from counselling when they need to consider a complicated problem, make an important decision (regarding contraception or HRT), adjust to changes in their lives (with ageing or after a diagnosis of ishaemic heart disease or diabetes is made) or think about changing their behaviour (giving up smoking, adopting a healthy lifestyle with exercise). In these situations counselling helps people to make decisions taking into account their circumstances and their goals.

Counselling is used when a person needs to consider a complicated problem, make an important decision, adjust to changes in their lives or think about changing their behaviour (adopting a healthy lifestyle with exercise). It is useful for a doctor to possess these counselling and communication skills.

Counselling is a communication process and doctors who are proficient in this communication processes are more likely to make accurate diagnoses, detect emotional distress in patients, have patients who are satisfied with their care and patients who agree with the advice given. In this way good communication helps the patient to make better health decisions. Therefore this skill is a valuable asset for doctors.

In a counselling relationship, the counsellor and client/patient work together to explore the patient's circumstances, enabling the individual to re-evaluate his or her experiences and potential. Counsellors facilitate full and confidential expression of the

\footnotetext{
${ }^{1}$ Consultant Psychiatrist, University Psychiatry Unit, National Hospital of Sri Lanka and Department of Psychological Medicine, Faculty of Medicine, University of Colombo, Sri Lanka.

Correspondence: Sunera M. Fernando

E-mail: @
}

patient's feelings, without diverting any attention to their own feelings. The counsellor is perhaps the first person that the individual has met for a long time who truly listens without prejudice and whom he or she can trust.

When counselling older persons it is important to remember that they may have hearing deficits, or cognitive deficits that may make them have difficulty understanding concepts.
For effective counselling
- Ensure privacy
- Seating arrangement
- Introduce yourself
- Qualify doubts
- Listen attentively

\section{Principles of counselling}

Before the counselling session make sure there is adequate privacy and the seating arrangement is satisfactory (the patient is seated at an angle to the interviewer). Putting the patient at ease before the interview is useful and this may be done by starting with easy questions. It is appropriate to begin the session by introducing yourself and greeting the patient by name. Listen actively to the patient's concerns by paraphrasing and encouraging the patient to talk. Clarify doubts and listen with enthusiasm and concern. Communication may be facilitated with nonverbal cues (eye contact, nodding head appropriately, leaning forward slightly) and verbal cues (saying 'yes I understand, please continue'). Using these skills a good therapeutic alliance can be created over the course of the session. This results in good rapport which is important to help the patient to achieve the therapeutic goals (for example adjust to changes in their lives, discuss new treatments or adopt healthy lifestyles).

In order to make the patient able to help him/her self during the session, the patient needs to feel safe enough to be open about their thoughts, feelings and behaviour. The patient also needs to feel respected and understood. Some of the skills that may be useful to the counsellor are given below. 
The counsellor must

\section{Listen}

Not judge

Pay attention

Accept the patient's feelings

Understand the patient's feelings and express that understanding

\section{The counsellor may}

Ask questions

Summarise

Ask the patient to try new behaviour

\section{Counsellors should not}

\section{Argue}

Dwell on their own difficulties

Solve the problem for the patient

Belittle the clients' concern

Avoid painful areas

\section{So that the patient can}

Develop his/her thinking

Feel safe and respected

Know you care

Know he/she is not being judged

Know you are with him/her

\section{So that the patient can}

Develop her/his own thinking

Hear her/his thoughts and know she/he is understood

Give confidence to him/ her and encourage him/her

\section{This will make the patient}

Defensive

Withdraw

Dependant

Withdraw or attack

Be frustrated

\section{Skills needed for a counselling session}

Good listening is an essential skill of communication and much more than just listening to the speaker.

\begin{tabular}{|l|}
\hline Counselling skills \\
Active listening \\
Body language \\
Open questions \\
Paraphrasing \\
Summarising \\
\hline
\end{tabular}

Active listening happens when you "listen for meaning". The listener says very little but conveys much interest by showing enthusiasm and concern. The listener only speaks to find out if a statement has been correctly heard and understood. Active listening is listening without passing judgement but reflecting back on what has been said to indicate that the feelings of the speaker have been understood. The listener may give neutral summaries to encourage the speaker. Active listening is hard work. The listener has to put in as much effort and energy into listening, as the speaker puts into speaking.

\section{What to avoid when counselling \\ Multiple questions \\ Irrelevant questions \\ Judgemental attitudes \\ Trying to hurry the interview (showing impatience)}

Body language takes into account our facial expressions, angle of our body, and proximity of ourself to another. Maintaining good eye contact is a good communication skill. Frequent eye contact shows 
the patient that you are interested in what they are saying. Patients may interpret poor eye contact as a sign of lack of interest in what they are saying. Make sure your body language is open, tone is friendly and calm to convey empathy and acceptance so that the patient will feel free to open up. You need to monitor the tone of your voice - in the same way that you monitor your body language. Remember, the person may not remember what was said, but they will remember how you made them feel!

Open and closed questioning are techniques that may be used in this communication process. An open question is one that is used in order to gathering lots of information - you ask it with the intent of getting a long answer. It is advisable to begin the session with open questions. A closed question is one used to gather specific information - it can normally be answered with either a single word or a short phrase. These questions are used to clarify specific points.

Paraphrasing is when you restate what the patient said in your own words. You may use paraphrasing to draw attention to a particular concern or aspect. Sometimes paraphrasing is used to clarify. Paraphrasing encourages the patient to talk and shows that you have understood what they have said.

Summarizing is focusing on the main points of a presentation or conversation in order to highlight them. At the same time you are giving the "gist", and checking to see if you are accurate.

Removing distractions: This is important for effective communication because distractions make the patient feel that you are not interested in what they are saying. It also shows the patient that you are not attentive and may be missing part of their message. There are a number of sources of distractions such as environmental factors (noise, telephones) and distraction within the doctor such as daydreaming and getting distracted by the patient's accent, mannerisms or language.

\section{Steps in giving information}

Giving information is also an effective communication skill. It can be used when advising patients on medication (HRT) or adopting healthy lifestyles. The following steps are important when giving information to facilitate the patient to make an informed decision on aspects of management:

- The content should be relevant and brief

- Give important information first

- Check patient's understanding of what has been explained

- Respond to patient's questions and emotional reactions

- Summarise the important issues for the patient

\section{Information giving}

- Relevant brief content

- Important information first

- Check patient's understanding

- Respond to questions

- Summarise

The clinical consultation is an important communication process. All the above communication skills may be incorporated into this interview to help to establish a good therapeutic relationship with the patient. The atmosphere created will show the patient that we have understood their perspective and problems. Effective communication skills can be used to formulate and share thoughts about the patient's illness and negotiate an action plan with the patient. In this way effective communication helps to facilitate the optimal healing environment.

\section{References}

1. Agarwal OP. Effective Communication, Global Media, 2010.

2. Ellis R. Communication Skills - Stepladders to Success for the Professional. Bristol, GBR: Intellect Ltd, 2002.

3. Hope S, Rees M. British Menopause Association, 2005. http:/ / www.thebms.org.uk/factdetail.php?id=4 accessed on 21 November, 2010. 